\title{
Adsorption-active polydisperse brush with tunable molecular mass distribution.
}

\author{
Anna S. Ivanova and Alexey A. Polotsky* \\ Institute of Macromolecular Compounds, Russian Academy \\ of Sciences. 31 Bolshoy pr, 199004 Saint Petersburg, Russia
}

Alexander M. Skvortsov

Chemical-Pharmaceutical University, Professora Popova 14, 197022 St. Petersburg, Russia

Leonid I. Klushin

Department of Physics, American University of Beirut. P. O. Box 11-0236, Beirut 1107 2020, Lebanon and
Institute of Macromolecular Compounds, Russian Academy
of Sciences. 31 Bolshoy pr, 199004 Saint Petersburg, Russia

Friederike Schmid

Institut für Physik, Johannes Gutenberg-Universität Mainz, Staudingerweg 9, D-55099 Mainz, Germany

(Dated: December 14, 2021)

\begin{abstract}
Recently a novel class of responsive uncharged polymer brushes has been proposed [Klushin et al, J. Chem. Phys. 154, 074904 (2021)] where the brush-forming chains have an affinity to the substrate. For sufficiently strong surface interactions, a fraction of chains condenses into a nearsurface layer, while the remaining ones form the outer brush with a reduced grafting density. The dense layer and the more tenuous outer brush can be seen as coexisting microphases. The effective grafting density of the outer brush is controlled by the adsorption strength and can be changed reversibly as a response to changes in environmental parameters.

In this paper we use numerical self-consistent field calculations to study this phenomenon in polydisperse brushes. Our results reveal an unexpected effect: Although all chains are chemically identical, shorter chains are adsorbed preferentially. Hence, with the increase in the surface affinity parameter, a reduction in the surface grafting density of the residual brush is accompanied by a change in the shape of its molecular mass distribution. In particular, an originally bidisperse brush can be effectively transformed into a nearly monodisperse one containing only the longer chain fraction. We introduce a method of assigning different chain conformations to one or the other microphase based on analyzing tail length distributions. In a polydisperse brush with a uniform molecular mass distribution (MMD) short chains are relegated to the adsorbed phase leading to a narrower effective MMD in the residual brush. Preferential adsorption is not absolute and longer chains are also partially involved in adsorption. As a result not only the width of the distribution decreases but its shape evolves away from the initial uniform distribution as well. We believe that the effect of preferential adsorption stems from a fundamental property of a polydisperse brush, which is characterized by a spectrum of chemical potential values for monomers belonging to chains of different length. Hence preferential adsorption is also expected in polyelectrolyte brushes; moreover, brush polydispersity would affect coexistence with any other condensed phase, not necessarily related to adsorption.
\end{abstract}

\section{INTRODUCTION}

Polymer brushes are commonly used for permanent surface modification to mediate the stability of colloidal dispersions, provide anti-fouling properties, and protect the system from degradation [1]. Brushes can also act as smart stimuli-responsive materials that change surface wetting properties reversibly or act as sensors [2, 3. Ways of manipulating brush properties in a reversible manner depend on the existing physical control parameters. In this respect, polyelectrolyte systems offer considerable flexibility since the interactions can be tuned, apart from the solvent quality, by changes in $\mathrm{pH}$ and the ionic strength of the medium as well as by applying an external electric field 4. In contrast, uncharged chemically homogeneous brushes seem to offer no obvious control "handles". Their physical properties under good solvent conditions are determined

* alexey.polotsky@gmail.com by two key parameters: the chain length and the grafting density. Both parameters are generally set at the brush synthesis stage and cannot be changed thereafter. Together they define the thickness of the brush layer and the strength of the repulsive forces that the brush exerts on objects approaching the surface.

Recently we investigated a novel class of responsive uncharged polymer brushes formed by endgrafted adsorption-active chains 5. In this case the brush properties are affected by the short-range adsorption interactions between the substrate and the chain units [6, 7]. It was demonstrated that a monodisperse brush with a strong enough monomer attraction to the substrate forms a microphase separated system with a fraction of chains being almost completely laid out on the surface while the rest of the chains form a residual brush with a reduced effective grafting density. As the adsorption energy $\varepsilon$ is increased, the fraction of chains in the adsorbed phase initially increases, but eventually the system saturates at large values of $\varepsilon \gtrsim 3 k T$. Phase coexis- 
tence is retained in a very broad range of $\varepsilon$, and it is impossible to identify a transition point. Indirectly, the adsorption parameter controls the grafting density $\sigma$ of the residual brush. Depending on the value of the product $\sigma N$ in the saturation limit, the residual brush may disappear completely or be reduced to isolated mushroom-like tails. We have identified and studied the regime, $\sigma N>2.5$, in which the residual brush is reasonably well defined for any value of the adsorption energy $\varepsilon$. The connectivity of the brush chains transmits a strong coupling between the adsorbed and desorbed layer. It is reflected in strong fluctuations of individual chains between "coexisting" adsorbed desorbed states, which are not sharply defined as the free energy barrier separating them is always small, less than $1 k T$ even when the adsorption energy is as large as $\varepsilon=10 k T$. This means that kinetic trapping is most likely absent, and the exchange of monomers between phases must be characterized by relatively fast dynamics.

Theoretical work on the response of polymer brushes to external variables is mostly based on monodisperse brush models 8, 9]. In real polymer systems polydispersity is almost unavoidable, although special methods for synthesizing monodisperse brushes have recently been introduced [10, 11. The most common method of brush synthesis based on the "grafting from" approach uses a surface immobilized initiator layer and subsequent in situ polymerization to generate the polymer brush. This method gives a polydisperse brush with the molecular weight distribution close to Schulz-Zimm distribution [12].

In this paper we investigate how polydispersity affects the properties of brushes formed by adsorptionactive chains. We will demonstrate that adsorption from a polydisperse brush exhibits an unexpected effect: Although all chains are chemically identical, shorter chains are adsorbed preferentially. As a result, an increase in the surface affinity parameter leads to a reduction in the surface grafting density of the residual brush, which is accompanied by a change in the shape of the molecular mass distribution. In order to establish the effect of preferential adsorption, we start with a detailed numerical self consistent field (SCF) investigation of a bidisperse brush. A subsequent study of a simple brush model with a flat continuous molecular mass distribution (MMD) confirms this effect and illustrates the changes in the MMD shape with the increase in the adsorption parameter.

\section{MODEL AND METHOD}

We consider two realizations of model polydisperse brushes: Bidisperse brushes and polydisperse brushes with a uniform chain length distribution (see Figure 1).

The bidisperse polymer brush is made of two types of linear flexible macromolecules differing only in the degree of polymerization grafted at one end onto a solid planar substrate. Polymer chains are com- (a)

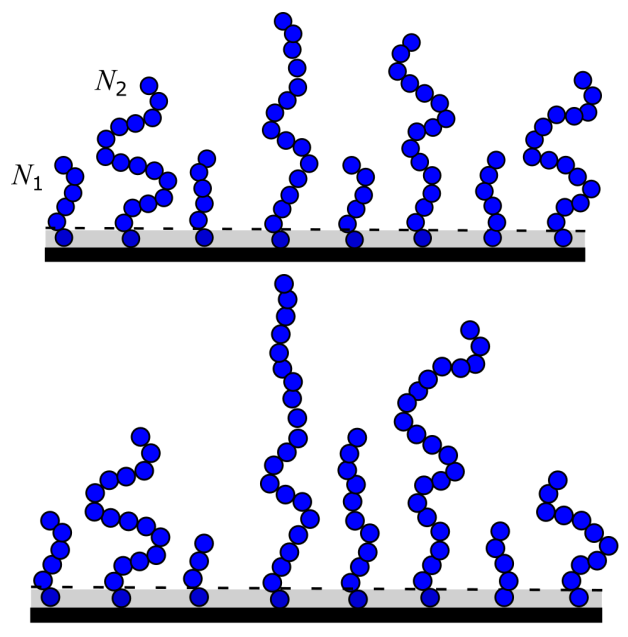

Figure 1. Cartoons of bidisperse (a) and polydisperse brushes.

posed of $N_{1}$ and $N_{2}>N_{1}$ identical monomer units, the number of short and long chains in the brush are equal. The average degree of polymerization is, therefore, $\bar{N}=\left(N_{1}+N_{2}\right) / 2$. The chains are grafted onto the surface at the grafting density $\sigma$, defined as the number of grafted polymer chains per unit surface area. The surface is assumed to be attractive to all polymer chains and the monomer-surface attraction is characterized by the adsorption energy $-\varepsilon, \varepsilon>0$. The brush is immersed into an athermal solvent; in terms of Flory-Huggins interaction parameter $\chi$, this corresponds to $\chi=0$.

In the uniform polydisperse brush, the chain length distribution is flat. This means that chains composed of $N=1,2,3, \ldots, N_{\max }$ monomer units are present in the brush, with the same probability equal to $p(N)=1 / N_{\max }$. This choice provides a proper normalization: $\sum_{N=1}^{N_{\max }} p(N)=1$. The average polymerization degree is

$$
\bar{N}=\sum_{N=1}^{N_{\max }} N p(N)=\frac{1+N_{\max }}{2} .
$$

Hence, to obtain a polydisperse ensemble of grafted chains with the desired $\bar{N}$, one should choose $N_{\max }=2 \bar{N}-1$. In particular, for $\bar{N}=100$ used in the present work, we have $N_{\max }=199$.

To calculate the system's partition function and its various properties, we use the Scheutjens-Fleer self-consistent field (SF-SCF) method. The SFSCF method and its modifications for the study of polymer brushes of various types have been repeatedly described in the literature and can be found in 8, 13, 14. The SF-SCF approach uses a lattice and also takes into account the geometry and the symmetry of the problem under consideration. For the planar polymer brush, the choice of the simple cubic lattice is obvious; polymer chains are modeled as walks on this lattice. The lattice cell size is equal to the size of a monomer unit, and each lattice site can be occupied either by a monomer unit by or a solvent molecule. The lattice sites are organized in planar layers, each layer is referred to 
with a coordinate $z$ normal to the grafting plane. Within a layer with fixed $z$, i.e., along $x$ and $y$ axes, the volume fractions of the monomeric components and the self-consistent potential are taken as uniform; hence, we use a one-gradient version of the SF-SCF method for planar geometry. A monomer unit in the first lattice layer adjacent to the surface has a contact with the surface and acquires an additional energy gain $-\varepsilon$. More details about the implementation of the SF-SCF method for the polymer brush with adsorption-active chains are given in Appendix of Ref. [5]. The modifications for bidisperse and polydisperse brushes are done according to [14. SF-SCF calculations were performed by using sfbox program developed in the Laboratory of physical and colloid chemistry at the University of Wageningen (the Netherlands).

\section{RESULTS}

\section{A. Bidisperse brush}

We start with a bidisperse brush made of two fractions of chemically identical polymer chains of lengths $N_{1}$ and $N_{2}$ and respective surface grafting densities $\sigma_{1}$ and $\sigma_{2}$, where index "1" refers to short chains, and index " 2 " refers to long ones so that $N_{1}<N_{2}$. We restrict our calculations to the case of equal fractional grafting densities $\sigma_{1}=\sigma_{2}=\sigma / 2$, where $\sigma$ is the total grafting density. As the basic system, we chose a brush made of chains that differ in length by 4 times: $N_{1}=40, N_{2}=160$. The number of short and long chains per unit area in the brush is the same, therefore the ratio of mass fractions of short chains to long ones is $1: 4$. At the same time, we will not restrict ourselves to only this set of chain lengths and will also consider the cases when the lengths of short and long chains differ less.

\section{The structure of bidisperse brush: polymer density profiles and free ends distributions}

The main characteristics of the polymer brush structure are the density profile and the free ends distribution. Figure 2 shows the evolution of the polymer density profile and the cumulative free ends distribution profile in the bidisperse brush upon a change in the energy $\varepsilon$ of the monomer units attraction to the grafting surface. As in monodisperse brushes, the polymer-surface attraction leads to an increase in polymer density in the first lattice layer adjacent to the surface. The brush as a whole becomes a little less dense and its thickness also decreases. The range of density variation in the first layer is much larger than in the rest of the brush, and for better visualization we show the density profiles on a smaller scale in the insets to Figures $2 \mathrm{a}$ and $2 \mathrm{~b}$.

In the distribution of free chain ends, Figure $2 \mathrm{c}, \mathrm{d}$, a typical curve has two peaks. In the case of an inert or very weakly attractive surface, these are familiar peaks corresponding to the ends of short and long chains. With increasing adsorption parameter, the proximal peak grows in magnitude and shifts to the first layer adjacent to the adsorbing surface, thus representing the adsorbed phase. The distal peak changes less and represents the residual brush.

The total profiles do not indicate clearly the chains of which type are preferentially adsorbed on the surface. To clarify this, we must consider the contributions of short and long chains to the total profiles separately. This is shown in Figure 3. We can see that the ends of the short and long chains are well separated at $\varepsilon=0$, the overlap of end distributions for short and long chains is small.

The curves unambiguously indicate that with increasing $\varepsilon$, short chains adsorb onto the grafting surface prior to the long ones, which only start adsorbing at stronger monomer-surface attraction. Accordingly, the most noticeable changes appear in the end profile $P_{e}(z)$ of short chains, while the change in the corresponding profile for long chains remains insignificant. However, at high adsorption strength one can see that the fraction of long chains also segregates into two microphases, similar to the case of a monodisperse brush [5]. Hence, we can draw here our first conclusion about the preferential adsorption of short chains on the grafting surface in a bidisperse brush.

\section{Adsorbed monomer fractions for short and long chains}

The relative fractions of monomers in the surface layer at $z=1$, where monomers feel the surface attractive potential, define the average adsorbed fractions for short and long chains:

$$
\left\langle\theta_{i}\right\rangle=\frac{2 \varphi_{i}(z=1)}{\sigma N_{i}}
$$

where $i=1$ stands for short chains and $i=2-$ for long chains.

Figure 4 shows that with increasing $\varepsilon$, the fractions of adsorbed units in short and long chains monotonically increase and reach saturation values. The fraction of adsorbed units in short chains is always larger than that in long chains thus pointing to the preference for the short chain adsorption in the brush. Lower values of $\langle\theta\rangle$ for the long chains correlate with the fact that they mostly belong to the residual brush phase as shown in the previous subsection. However, the maximum of the average fraction of adsorbed units in short chains does not reach unity indicating some competition for adsorption with the long chain fraction. An increase in the grafting density leads to a decrease in $\left\langle\theta_{i}\right\rangle$ for both short and long chains simply because the adsorbing capacity of the surface is limited and more chains belong to the residual brush. At the largest grafting density, $\sigma=0.1$, the sub-brush formed by short chains is relatively dense and the long chain fraction is almost completely excluded from adsorption.

Preferential adsorption is due to the difference in the chain length between the two chain fractions. 

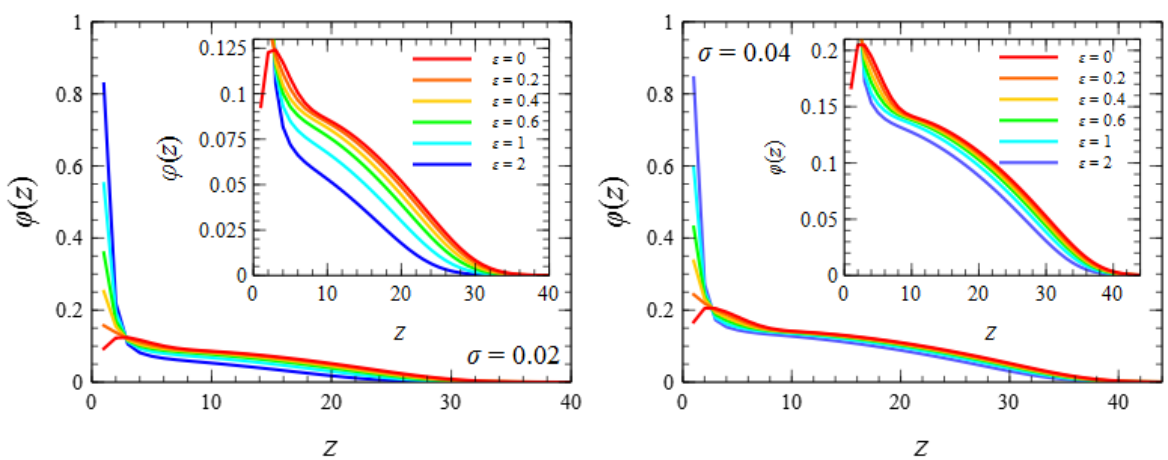

(a)
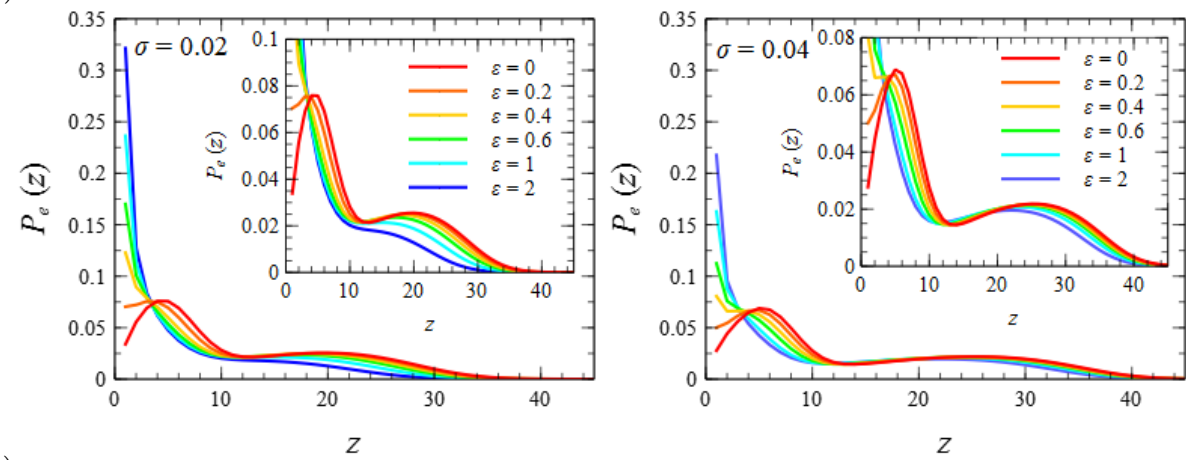

(b)

(c)

Figure 2. Density profiles (monomer volume fraction) $\varphi(z)(\mathrm{a}, \mathrm{b})$ and end monomer density profiles $P_{e}(z)$ (c, d) for an adsorption-active bidisperse brush made of polymer chains of lengths $N_{1}=40$ and $N_{2}=160$ with equal fractional grafting densities; total surface grafting density $\sigma=0.02(\mathrm{a}, \mathrm{c})$ and $\sigma=0.04(\mathrm{~b}, \mathrm{~d})$; surface adsorption energy $\varepsilon$ values are indicated in the graphs. Insets show the profile details at smaller $\varphi$ and $P_{e}$ scales.
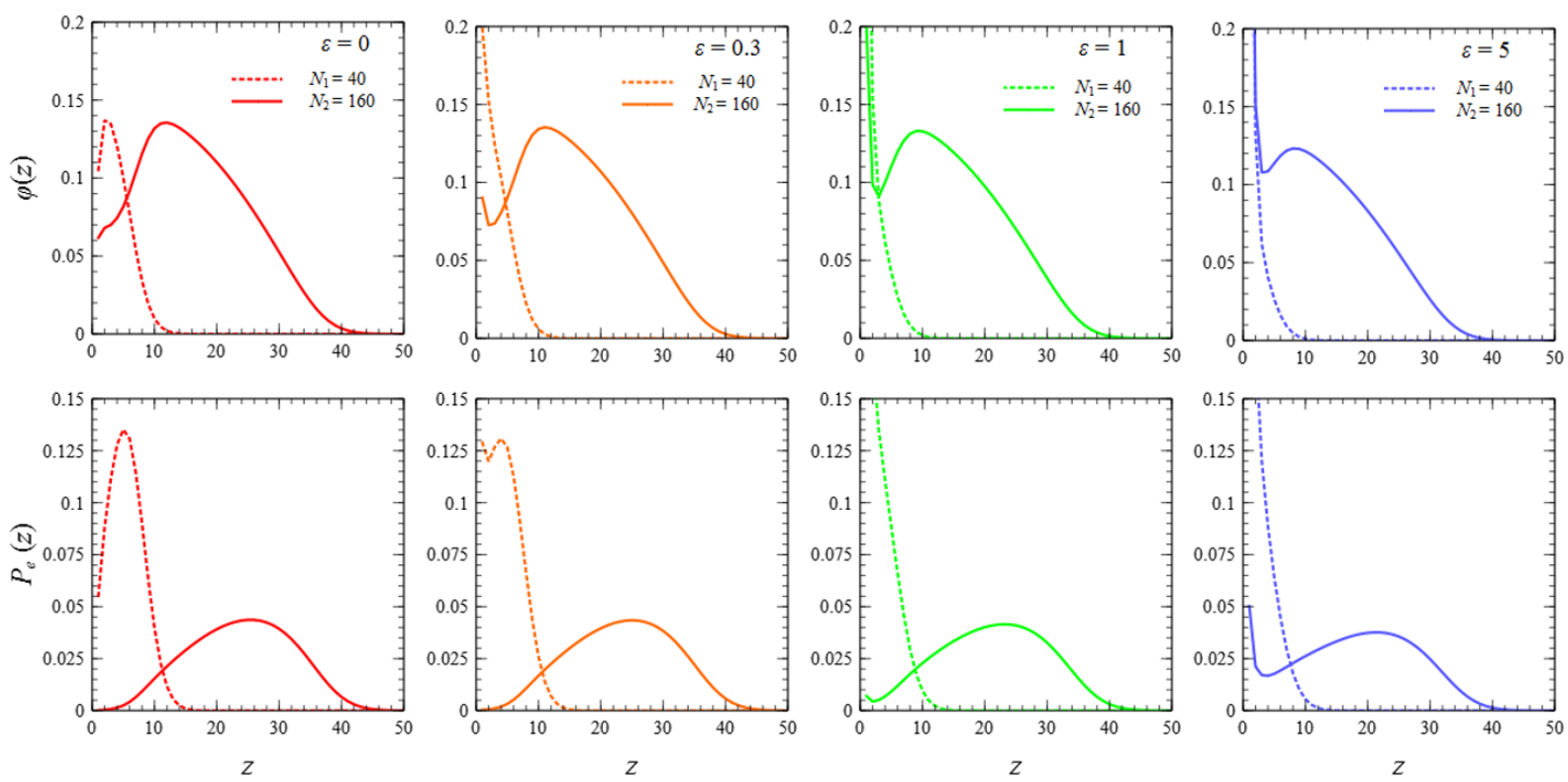

Figure 3. Short (dashed lines) and long (solid lines) chain contributions to the brush density profiles $\varphi(z)$ (upper row) and free end density profiles $P_{e}(z)$ (lower row) in a bidisperse brush with $N_{1}=40$ and $N_{2}=160$ and total surface grafting density $\sigma=0.02$, at various values of the polymer-surface adsorption energy $\varepsilon$ as indicated.

We expect the adsorption curves for the two chain fractions to come closer and eventually merge as the chain length difference becomes less, which is illustrated in Figure 5a. Figure 5b shows another aspect of the same picture: we plot the fraction of adsorbed units for short and long chains vs. the respective chain lengths, $N_{1}$ and $N_{2}$, keeping both $\varepsilon$ and the average molecular weight $\bar{N}=\left(N_{1}+N_{2}\right) / 2$ fixed. The fraction of adsorbed units in short chains demonstrates a monotonic decrease with increasing $N_{1}$, consistent with Figure 5 a. One could expect that the fraction of adsorbed units in long chains, $\left\langle\theta_{2}\right\rangle$, will exhibit a matching behaviour - a monotonic increase with the decrease in the length of the long chain, $\mathrm{N}_{2}$, i.e. upon approaching the monodisperse situation. However, it shows a non-monotonic 


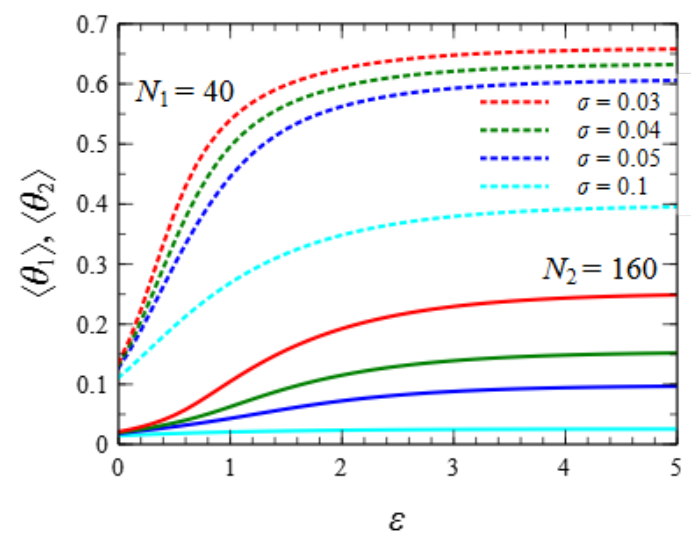

Figure 4. Average fraction of adsorbed monomers in short chains $\left\langle\theta_{1}\right\rangle$ (dotted lines) and in long chains $\left\langle\theta_{2}\right\rangle$ (solid lines) in a bidisperse brush with $N_{1}=40$ and $N_{2}=160$ as a function of the adsorption energy $\varepsilon$; the total grafting density $\sigma$ is indicated in the Figure.

behaviour at $\varepsilon>0$ : at small $N_{1}$, it decreases with increasing $N_{1}$, passes through a minimum and then starts to increase with $N_{1}$, as expected.

A tentative explanation of such a non-monotonic behavior is suggested by a close look into the structure of the brush in the case of very strong attraction and small $N_{1}$. Assuming for simplicity full preference for short chain adsorption, the chain needs $N_{1}$ surface sites to be fully adsorbed. The surface area per chain in the brush is equal to $1 / \sigma$, that is, there are $1 / \sigma$ surface sites (lattice cells in the first layer), or vacancies, that can be occupied. Since we have an equal number of short and long chains in the brush, the number of adsorption sites per short chain is $2 / \sigma$. Under the condition $N_{1}<N_{1}^{*}=2 / \sigma$, all short chain can be (in principle) fully adsorbed. The remaining $N_{1}^{*}-N_{1}$ sites can be occupied by long chain monomers. Assuming that the long chains occupy all these remaining sites, the average fraction of adsorbed units per long chain is

$$
\left\langle\theta_{2}\right\rangle=\frac{N_{1}^{*}-N_{1}}{N_{2}}=\frac{N_{1}^{*}-N_{1}}{2 \bar{N}-N_{1}}=1-\frac{2 \bar{N}-N_{1}^{*}}{2 \bar{N}-N_{1}} .
$$

Then

$$
\frac{d\left\langle\theta_{2}\right\rangle}{d N_{1}}=-\frac{2 \bar{N}-N_{1}^{*}}{\left(2 \bar{N}-N_{1}\right)^{2}}
$$

is negative and $\left\langle\theta_{2}\right\rangle$ is thus clearly a decreasing function of $N_{1}$. Note that the assumption of full adsorption of all short chains included in Eq. (3) is a simplification: in the full picture there is always some competition for surface sites between short and long chains. Even within the simplified picture, full adsorption of short chains only can take place for $N_{1}<N_{1}^{*}$, while for $N_{1}>N_{1}^{*}$ the "adsorption capacity" of the grafting surface is not sufficient. At the same time, as $N_{1}$ grows beyond $N_{1}^{*}$, the length difference between the long and short fractions starts decreasing and the competition becomes more pronounced, thus leading to a growth in $\left\langle\theta_{2}\right\rangle$.

\section{Tail length distribution as an indicator of phase} coexistence

The concept of a tail appears in polymer adsorption studies as an element of the adsorbed chain conformation. By definition, the tail of an adsorbed chain in a lattice model is the subchain that starts in the layer $z=2$, next to the surface layer $z=1$, and never returns to the surface layer at $z=1$. Naive cartoons of brushes grafted to an inert surface invariably suggest that all brush chains have tail conformation (see Figure 1 as an example). This would imply that in a polydisperse brush, the tail length distribution coincides with the MMD of the brush chains. This is not quite true, as the monomer density in the first layer $\propto \sigma^{2 / 3}$ is considerably larger than the density of grafting points $\propto \sigma$, and hence some parts of the brush chains form loops.

Figure 6 shows the evolution of the tail length distribution in a bidisperse brush with increasing polymer-surface attraction. In the case of a neutral surface, $\varepsilon=0$, two peaks corresponding to the two chain fractions are observed; however, the shape of the tail distribution is not identical with the bare chain length distribution, which consists of two delta peaks in a bidisperse brush. With increasing attraction strength, the peak heights at $s=N_{1}-1$ and $s=N_{2}-1$ both decrease, the distribution width corresponding to the long chain fraction increases. The width of the short fraction contribution changes only little, but a secondary peak describing vanishingly small tails appears and eventually becomes dominant. It is natural to associate the vanishing tail peak with the adsorbed microphase. The evolution of the tail length distributions is consistent with the notion of preferential adsorption of short chains.

To get an additional insight into preferential adsorption, we compare the distribution of short-chain and long-chain tails in a bidisperse brush with the distributions of tails in monodisperse brushes. Two types of reference monodisperse brushes can be constructed: 1) all the chains of the other fraction are replaced by chains of the fraction of interest, meaning that $\sigma_{\text {mono }}=\sigma_{b i} ; 2$ ) all the chains of the other fraction are removed without replacement, meaning that $\sigma_{\text {mono }}=1 / 2 \sigma_{b i}$. Figures 7 and 8 show this comparison for the long and the short fractions, respectively.

For the long chain fraction, see Figure 7 the picture is closer to the tail length distribution in a monodisperse brush with the same total grafting density, $\sigma_{m o n o}=\sigma_{b i}$. On the other hand, Fig. 8 indicates that the situation regarding the adsorption of the fraction of short chains is more complex: At small adsorption strength $\varepsilon$, it seems little affected by the presence of the long chains, and the reference monodisperse brush with $\sigma_{\text {mono }}=1 / 2 \sigma_{b i}$ is more appropriate. However, as $\varepsilon$ increases and the long chains gradually start adsorbing as well, the tail length distribution differs substantially from those in both reference systems. 

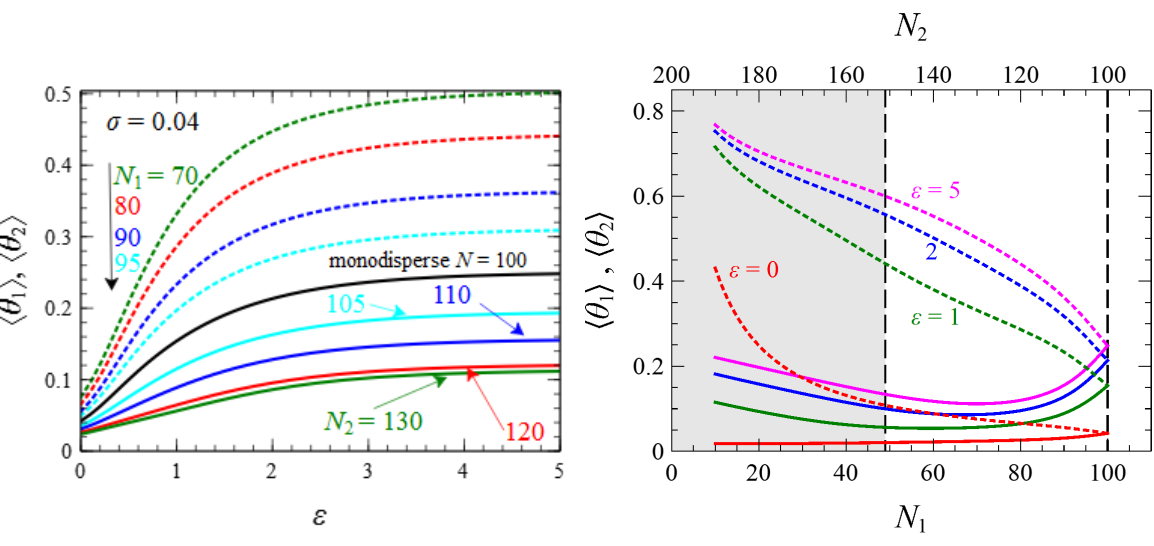

(a)

(b)

Figure 5. Average fraction of adsorbed monomers in short chains $\left\langle\theta_{1}\right\rangle$ (dotted lines) and in long chains $\left\langle\theta_{2}\right\rangle$ (solid lines) in a bidisperse brush with average chain length $\bar{N}=\left(N_{1}+N_{2}\right) / 2=100$ as a function of the adsorption energy $\varepsilon$ for different chain length differences $\left(N_{1}-N_{2}\right)$ as indicated (a); same quantities vs. the respective chain lengths $N_{1}$ (lower scale) and $N_{1}$ (upper scale) (at fixed $\bar{N}=100$ ) for different values of the adsorption energy $\varepsilon$ as indicated (b). The total grafting density is $\sigma=0.04$ in both graphs. The vertical dashed line at $N_{1}^{*}=2 / \sigma$ in Fig. (b) separates two regimes differing in the ability of short chains to cover the adsorbing surface, see discussion in the text.

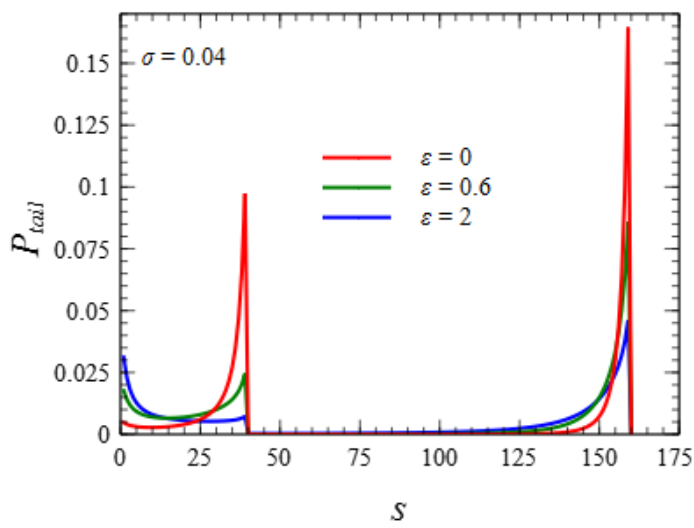

Figure 6. Tail length distribution in a bidisperse brush with $N_{1}=40$ and $N_{2}=160$ and total surface grafting density $\sigma=0.04$ at various values of the polymer-surface adsorption energy $\varepsilon$, as indicated.

\section{Quantifying phase coexistence through tail length distribution}

In our previous work [5], we studied a monodisperse brush composed of adsorption-active chains and considered two possible adsorption scenarios: (1) partial adsorption of all chains or (2) complete adsorption of only part of the chains. The results of numerical calculations by the Scheutjens-Fleer SCF method and scaling arguments have proved the second scenario to be a more adequate picture of the phenomenon. The tail length distributions that were introduced above provide yet another direct evidence supporting this conclusion. Indeed, partial adsorption of all chains would have resulted in a unimodal distribution with a maximum at intermediate tail length shifting to the left with the increase in the adsorption strength. On the other hand, bimodal distributions are consistent with the second scenario. In a bidisperse brush the situation is complicated by the presence of two distinct chain fractions with a clear indication of preferential adsorption for short chains. As a result we get a residual bidisperse brush with a reduced overall grafting density and a different effective composition. In [5], we have proposed a fitting procedure based on comparing the density profiles of an adsorption-active monodisperse brush with those in non-adsorbing brushes at reduced grafting densities that allowed us to quantify the effective grafting density of the residual brush, see Figure 9(a) that illustrates this approach. We can extend this procedure to bidisperse brushes at the cost of going to a two-dimensional space of fitting parameters. Sadly, for polydisperse brushes this cost becomes impossibly high.

On the other hand, our analysis shows that there exists another way of determining the relative weights of the adsorbed and the brush phases, based on the tail length distribution. The idea is that the tail length distribution in the monodisperse brush is bimodal, with maxima at two boundaries $s=1$ (the shortest possible tail) and $s=N-1$ (the longest possible tail), see Figure 9(b). With increasing $\varepsilon$, the maximum corresponding to a vanishing tail becomes more prominent both in terms of height and of the integrated overall weight. The two maxima are separated by a shallow and broad minimum. We take the position of the minimum as the boundary separating the conformations belonging to the adsorbed and brush phases. This definition is based on reasoning that the chains in the adsorbed phase have predominantly short tails, whereas the chains in the brush phase have long tails essentially coinciding with the brush-forming chains themselves.

The definition we adopt forces us to assign some small but non-zero weight to the adsorbed phase even in the absence of explicit attractive potential, i.e. at $\varepsilon=0$, see the red curve in Figure 9(b). Although counter intuitive, it is consistent with two established facts: i) the monomer density in the first layer $\varphi(z=1) \sim \sigma^{2 / 3}$ is considerably larger than 

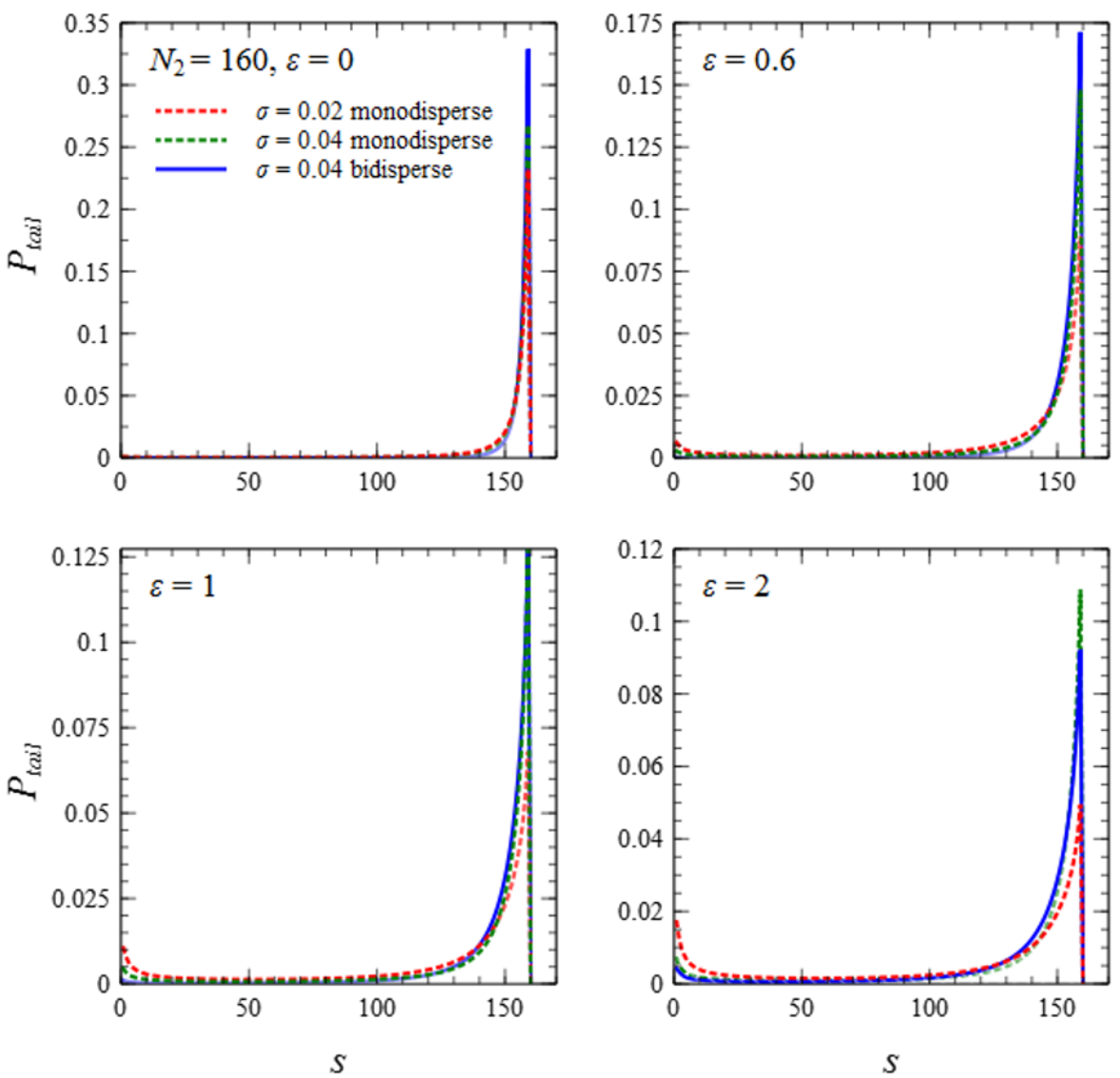

Figure 7. Tail length distribution for long chains $\left(N_{2}=160\right)$ in a bidisperse brush with $N_{1}=40$ and $N_{2}=160$ and total surface grafting density $\sigma=0.04$ (blue solid lines) in comparison to reference monodisperse brushes with $N=N_{2}=160$ at grafting density $\sigma=0.04$ (red dotted lines) and $\sigma=0.02$ (green dotted lines) at various values of the polymer-surface adsorption energy $\varepsilon$, as indicated.

the contribution, $\sigma$, of the grafting monomers themselves meaning that there are of order $\sigma^{-1 / 3}$ contacts with the inert impenetrable surface per chain; ii) the self-consistent brush density profile has a small dip in the nearest proximity of the grafting surface, which generates an osmotic force pushing monomers towards the surface, hence an effective attraction: simple estimates show that its strength is of the order of the critical adsorption threshold.

According to this idea, we define the fraction of chains in the brush phase as

$$
q=\sum_{s=s_{m i n}+1}^{N-1} P_{\text {tail }}(s)
$$

where $s_{\min }$ is the tail length corresponding to the minimum of the distribution. We attribute the tails with $s_{\text {min }}$ monomer units to the adsorbed phase and, therefore start summation in Eq. (5) from $s=s_{\min }+1$. The fraction of chains in the adsorbed phase is $1-q$ and this also includes chain conformations with no tails $(s=0)$. The fraction of chains belonging to the brush phase can be also understood as the effective grafting density of the residual brush reduced by the grafting density at brush preparation:

$$
q=\frac{\sigma_{\text {eff }}}{\sigma}
$$

In Figure 10 we compare two ways of calculating the ratio $\frac{e_{e f f}}{\sigma}$ for monodisperse brushes with $N=100$ and obtain a very satisfying agreement. Hence, we can conclude that the tail length distribution gives us a simple and robust way of quantifying the relative weights of the adsorbed and the brush phases. This method does not require a fitting procedure and can be easily generalized to bidisperse and polydisperse brushes.

A further comparison of the application of the two methods to bidisperse brushes is displayed in Figures 11 and 12. In contrast to the monodisperse case, one has to define the relative weights of the adsorbed and brush phases for each chain fraction separately in view of the short chain preferential adsorption of as mentioned above. The normalized effective grafting densities $q_{1,2}=\frac{\left(\sigma_{\text {eff }}\right)_{1,2}}{\sigma_{1,2}}=\frac{2\left(\sigma_{\text {eff }}\right)_{1,2}}{\sigma}$ are shown as functions of the adsorption parameter, $\varepsilon$, separately for short ( $i=1$, lower set of curves) and long ( $i=2$, upper set of curves) chains. Results obtained from fitting density profiles to equivalent brushes are shown by symbols, while results from integrating tail length distributions - by solid 

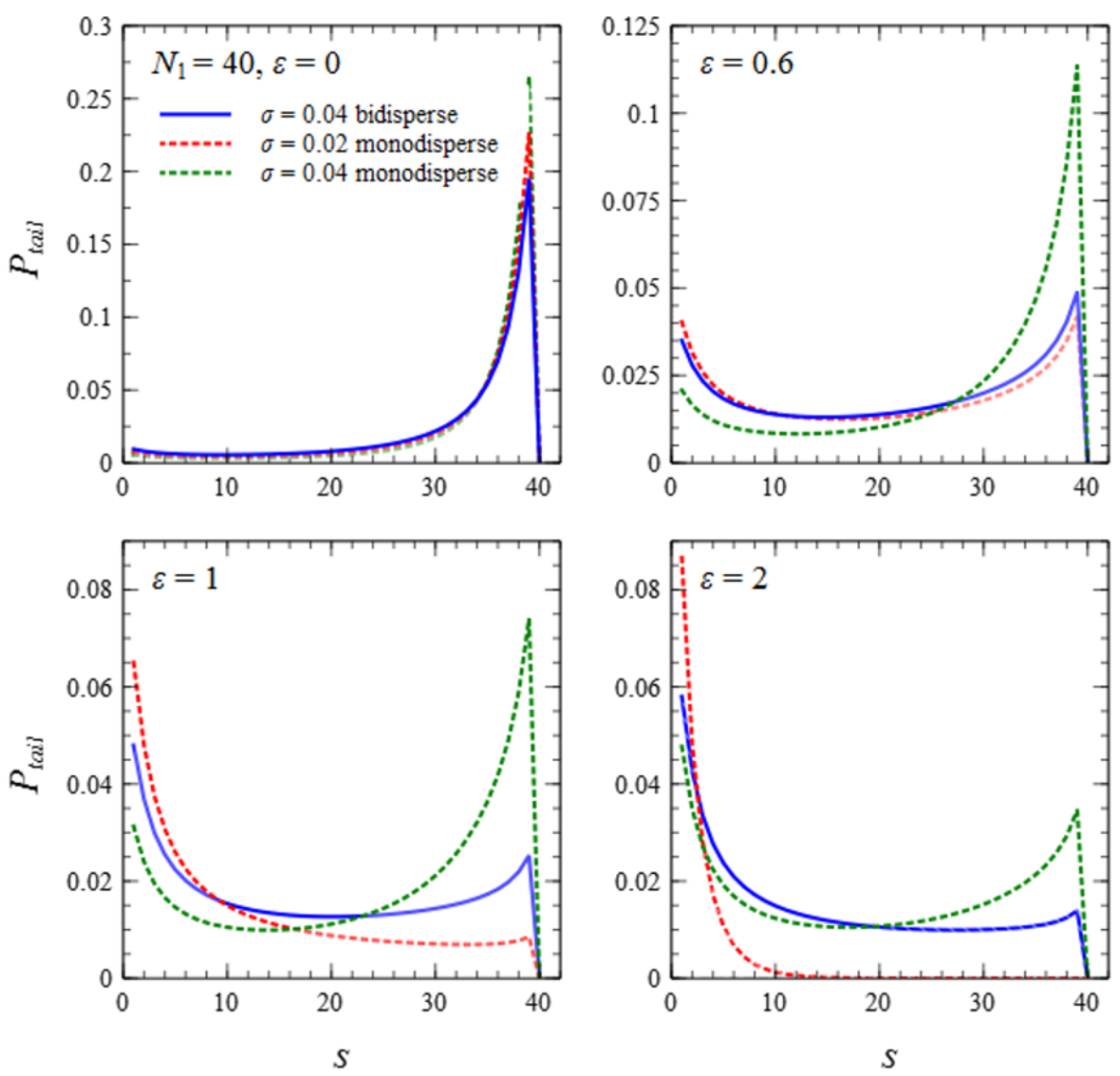

Figure 8. Tail length distribution for short chains $\left(N_{1}=40\right)$ in a bidisperse brush with $N_{1}=40$ and $N_{2}=160$ and total surface grafting density $\sigma=0.04$ (blue solid lines) in comparison to reference monodisperse brushes with $N=N_{1}=40$ at grafting density $\sigma=0.04$ (red dotted lines) and $\sigma=0.02$ (green dotted lines) at various values of the polymer-surface adsorption energy $\varepsilon$, as indicated.
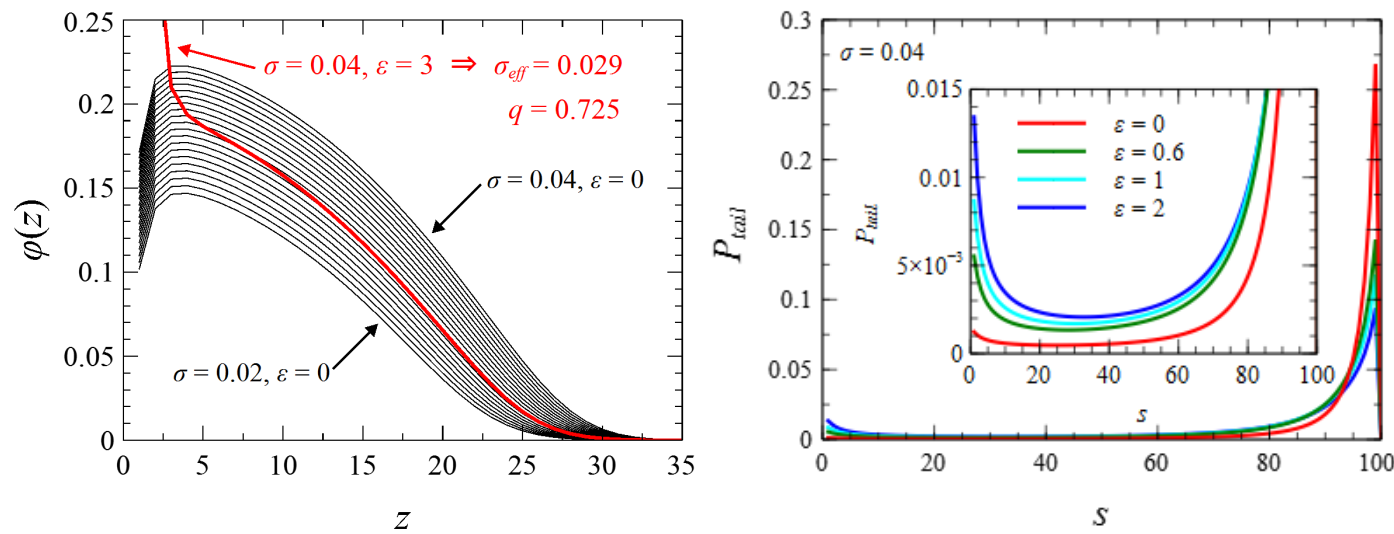

(a)

Figure 9. Illustration of the two methods of defining the effective grafting density of the residuals brush: comparing the density profile of an adsorption active brush with those of non-adsorbing brushes at reduced grafting densities (a); tail length distributions at various values of the polymer-surface adsorption energy $\varepsilon$, as indicated (b); the position of the minimum of the distribution is used to separate conformations as belonging to the adsorbed and the brush phases. All brushes are monodisperse with $N=100$, the grafting density of the active brush is $\sigma=0.04$.

lines. The effective grafting density of the residual brush decreases with increasing polymer-surface attraction as expected. Preferential adsorption of shorter chains is also clear. The tail length distribution method attributes a small fraction of the short chains to the adsorbed phase even at $\varepsilon=0$ as discussed above. For larger grafting densities, $\sigma$, the relative effect of the residual brush thinning as quantified by $\frac{\sigma_{\text {eff }}}{\sigma}$ is less pronounced for both short and long chains simply because the total adsorption capacity remains roughly the same while the normalizing constant increases. When the long and the short chains have comparable lengths the two methods give very close results, see Figure 11 b. There 


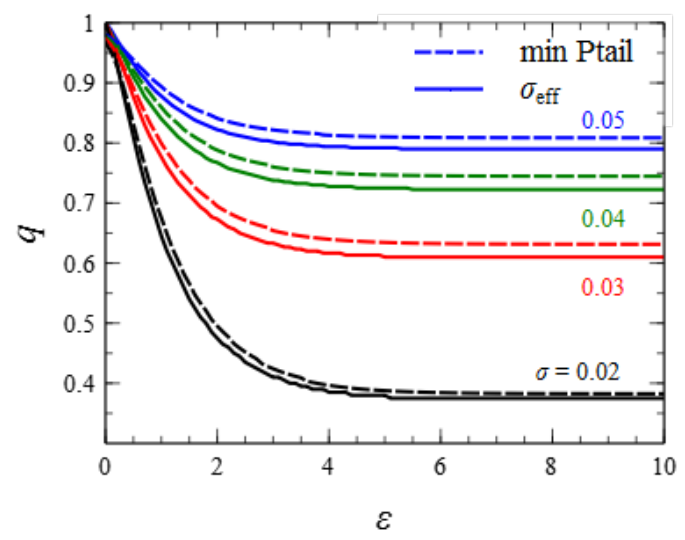

Figure 10. Effective fraction of chains in the residual brush in a monodisperse brush with $N=100$ as a function of the polymer-surface adsorption energy $\varepsilon$ and grafting densities $\sigma$ as indicated. Solid lines show the data calculated from the tail length distribution according to Eq. (5). Dashed line show the result obtained in [5] by fitting the polymer density profile to the density profile of the equivalent brush with reduced grafting density
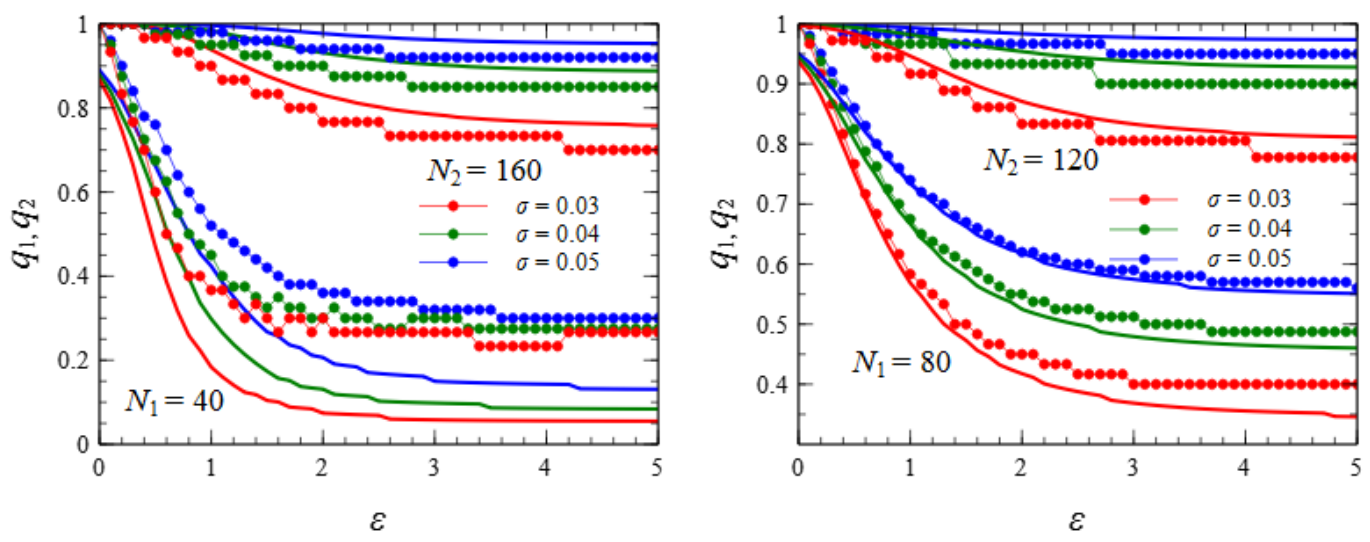

(a)

Figure 11. Normalized effective grafting densities $q_{1,2}=\frac{2\left(\sigma_{\text {eff }}\right)_{1,2}}{\sigma}$ of short and long chains in the residual brush
as functions of the polymer-surface adsorption energy $\varepsilon$ in bidisperse brushes with $N_{1}=40$ and $N_{2}=160$ (a) and $N_{1}=80$ and $N_{2}=120$ (b) at three total grafting densities $\sigma$ as indicated. Solid lines show the data calculated from the tail length distribution. Symbols show the result obtained by fitting the brush polymer density profile to the density profile of the equivalent brush of nonadsorbing chains with reduced partial grafting densities.

is a moderate discrepancy between the methods in the case of $N_{1}=40$ and $N_{2}=160$ when the overlap of the short chains is weak. Figure 12 shows that the preferential adsorption effect remains pronounced even when the relative length difference between the long and the short chains is quite low. Naturally, as $N_{1}-N_{2} \rightarrow 0$, preferential adsorption disappears and the two curves collapse into a single curve characterizing the monodisperse brush.

When the preferential adsorption is strongest ( $N=40$ and $\left.N_{2}=160\right)$ the short fraction is almost completely relegated to the adsorbed phase for $\varepsilon>2$ which means that the residual brush becomes effectively monodisperse.

\section{The origins of preferential adsorption}

We have demonstrated in Figure 12 that preferential adsorption is observed even when the relative length difference between the long and the short chains is as low as $\pm 5 \%$ as counted from the mean value. This is very different from finite-size effects in single-chain adsorption 8. Hence it must be related to the properties of bidisperse brush acting as a reservoir for the adsorbed phase.

To put this on a more quantitative basis, we first recall three important results of our previous work on monodisperse brushes [5]. First, the scaling analysis suggests that in the microphase separated regime, the substrate is covered by a dense layer of adsorption blobs of thickness $D \sim \varepsilon^{-\nu / \phi}$, containing $m_{a} \sim D^{1 / \nu} / D^{2} \sim \varepsilon^{-(1-2 \nu) / \phi}$ monomers per area. Here $\nu \sim 3 / 5$ is the Flory exponent and $\phi$ the crossover exponent 15, which is close to $\phi \sim 1 / 2$ according to numerical simulations [16 18. Importantly, $m_{a}$ does not depend on brush characteristics such as chain length and grafting density. Likewise, the free energy per area can be separated into an adsorption contribution and a brush contribution $F=F_{\text {ads }}+F_{\text {brush }}$, where the adsorption contribution $F_{\text {ads }} \sim-\varepsilon^{2 \nu / \phi}$, again, only depends on the adsorption strength $\varepsilon$. The arguments presented in Ref. 5] can be repeated for bidisperse chains, essen- 


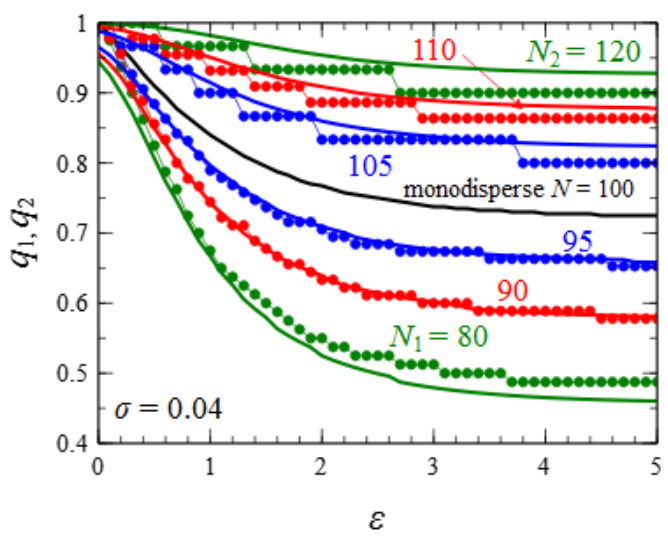

Figure 12. Normalized effective grafting densities $q_{1,2}=\frac{2\left(\sigma_{\text {eff }}\right)_{1,2}}{\sigma}$ of short and long chains in the brush phase as functions of the polymer-surface adsorption energy $\varepsilon$ in a bidisperse brush made of polymer chains with $N_{1}$ and $N_{2}$ monomer units as indicated grafted at fixed total grafting density $\sigma=0.04$. Solid lines show the data calculated from the tail length distribution. Symbols line show the result obtained by fitting the brush polymer density profile by the density profile of the equivalent brush of nonadsorbing chains with reduced grafting density.

tially leading to the same result. Second, the scaling analysis and the SCF calculations also indicated that chains either adsorb as a whole, or desorb as a whole, intermediate states with partly adsorbed chains are not favored. Third, SCF calculations of a single probe chain in the brush potential showed that the adsorbed and the desorbed state are well-defined and separated by an effective potential barrier.

Motivated by these findings, we now discuss the properties of an adsorption-active polydisperse brush made of chains with $K$ different chain lengths $N_{i}\left(i=1 \ldots K, N_{i+1}>N_{i}\right)$ and corresponding partial grafting densities $\sigma_{i}$ (with $\sigma=\sum_{i} \sigma_{i}$ ). We assume that a fraction $f_{i}$ of chains with length $N_{i}$ is adsorbed, where $f_{i}$ will be determined below. The total number of monomers per unit area is given by $m=\sum_{i=1}^{K} \sigma_{i} N_{i}$, and the number of monomers in the brush phase is $m-m_{a}$. The set of adsorbed fractions $\left\{f_{i}\right\}$ is thus subject to the constraint

$$
\sum_{i} \sigma_{i} f_{i} N_{i}=m_{a}=c \varepsilon^{(2 \nu-1) / \phi} .
$$

with the unknown prefactor $c$. In the scaling picture, the brush consists of $K+1$ layers (see Figure 13): The adsorption layer with thickness $D$ and $K$ brush layers with effective grafting densities $\widehat{\sigma}_{j}=$ $\sum_{i=j}^{K} \sigma_{i}\left(1-f_{i}\right)$, height $h_{j} \sim \widehat{\sigma}_{j}^{1 / 3}\left(N_{j}-N_{j-1}\right)$, and partial brush energies $F_{j} \sim\left(N_{j}-N_{j-1}\right) \widehat{\sigma}_{j}^{(2 \nu+1) / 2 \nu}$. Hence we get the brush energy

$$
F_{\mathrm{brush}}=C \sum_{i=1}^{K} \widehat{\sigma}_{j}^{X}\left(N_{j}-N_{j-1}\right) \quad \text { with } N_{0}:=0
$$

where $X=\frac{2 \nu+1}{2 \nu}=11 / 6$ and $C$ is an unknown prefactor. This corresponds nicely with the analytical result for the free energy of a bidisperse brush at $\varepsilon=0$ in the strong stretching limit [19]

$$
F_{\text {brush }}=\frac{9}{10}\left(\frac{\pi}{2}\right)^{2 / 3}\left(\sigma_{1}+\sigma_{2}\right)^{5 / 3} N_{1}+\frac{9}{10}\left(\frac{\pi}{2}\right)^{2 / 3} \sigma_{2}^{5 / 3}\left(N_{2}-N_{1}\right.
$$

except that the exponent $X$ is replaced by its meanfield value, $5 / 3$, which is slightly smaller than $X$ due to the neglect of excluded volume interactions [20, 21. Since every chain can assume two states, the free energy per area has an additional entropic contribution

$$
F_{\text {two-state }}=\sum_{i=1}^{K} \sigma_{i}\left[f_{i} \ln f_{i}+\left(1-f_{i}\right) \ln \left(1-f_{i}\right)\right],
$$

where we have set $k_{B} T=1$.

To calculate the fractions $f_{i}$, we must minimize

$$
I\left[f_{i}\right]=F_{\mathrm{ads}}+F_{\mathrm{brush}}+F_{\text {two-state }}+\lambda \sum_{i}\left(\sigma_{i} f_{i} N_{i}-m_{a}\right)
$$

with respect to $f_{i}$. Here the last term accounts for the constraint (7) with a Lagrange parameter $\lambda$, the free energy contributions $F_{\text {brush }}$ and $F_{\text {two-state }}$ are given by Eqs. (8) and (10), respectively, and $F_{\text {ads }}$ and $m_{a}$ do not depend on $f_{i}$ as explained above. The minimization equations $\partial I / \partial f_{i}=0 \forall i$ finally give the following expressions for the adsorbed fractions $f_{j}$ :

$$
f_{j}=1 /\left(1+\exp \left(N_{j}\left(\lambda-u_{j}\right)\right)\right)
$$

$$
\text { with } \quad N_{j} u_{j}=X C \sum_{i=1}^{j}\left(N_{i}-N_{i-1}\right) \widehat{\sigma}_{i}^{X-1} .
$$

Assuming that monomers in the adsorbed layer carry a free energy $e_{a}=F_{\text {ads }} / m_{a} \sim-\varepsilon^{-6 / 5}$, this corresponds to a Fermi-Dirac distribution for monomers with "temperature" $1 / N_{j}$, "energy" $e_{a}$ and "chemical potential" $\mu_{j}=u_{j}-\lambda-e_{a}$. The global parameter $\lambda$ sets the average monomer number in the adsorbed layer, which may fluctuate due to the coupling to the brush. The "chemical potential" $\mu_{j}$ can be interpreted as a potential driving the monomers into the adsorbed phase. It is related to the brush free energy penalty per monomer $u_{j}$ for chains of length $N_{j}$. Since longer chains stretch further out into regions where stretching penalties are low, $\mu_{j}$ decreases with increasing chain length $N_{j}$, as can easily be verified by calculating

$$
\begin{aligned}
\frac{\mu_{j}-\mu_{j+1}}{x C}=\frac{u_{j}-u_{j+1}}{X C} & =\left(1-\frac{N_{j}}{N_{j+1}}\right)\left(\frac{1}{N_{j}} \sum_{i=1}^{j}\left(N_{i}-N_{i-1}\right)\right. \\
& >\left(1-\frac{N_{j}}{N_{j+1}}\right)\left(\widehat{\sigma}_{j}^{X-1}-\widehat{\sigma}_{j+1}^{X-1}\right)>0
\end{aligned}
$$

where we have used that $\widehat{\sigma}_{j}$ decreases with $j$. This means that the free energy of the brush is reduced more efficently when expelling a monomer belonging to a short chain. 
In the extreme case of infinite chain length, $N_{j} \rightarrow$ $\infty$, the Fermi-Dirac distribution turns into a step function

$$
f_{j} \approx\left\{\begin{array}{lll}
1 & : & u_{j}<\lambda \\
f & : & u_{j}=\lambda \\
0 & : & u_{j}>\lambda
\end{array}\right.
$$

where $\lambda$ and $f$ must be chosen such that the constraint, Eq. (7), is fulfilled. Short chains (with larger $u_{j}$ ) adsorb first. An adsorption of chains of a certain length $N_{j}$ can only set in once all shorter chains have completely been removed from the residual brush.

Applying these results to the bidisperse case, we must distinguish between two partially adsorbed regimes in the limit of infinite chain length: (i) partially adsorbed short chains $\left(0 \leq f_{1} \leq 1\right)$, fully desorbed long chains $\left(f_{2}=0\right)$ and (ii) fully adsorbed short chains $\left(f_{1}=1\right)$, partially adsorbed long chains $\left(0 \leq f_{2} \leq 1\right)$. The constraint (7) gives $f_{1}(\varepsilon)=\frac{c}{\sigma_{1} N_{1}} \varepsilon^{-2 / 5}$ in the regime (i) and $f_{2}(\varepsilon)=$ $\frac{c}{\sigma_{2} N_{2}} \varepsilon^{-2 / 5}-\frac{\sigma_{1} N_{1}}{\sigma_{2} N_{2}}$ in the regime (ii), with a transition between regimes at $\varepsilon^{*}=\left(\sigma_{1} N_{1} / c\right)^{5 / 2}$. This is roughly consistent with the shape of the curves in Fig. 4, if one takes into account the smoothening of the curves at finite chain length.

In the case of a polydisperse brush with continuous MMD $P(N)$ (with $\int \mathrm{d} N P(N)=1$ ), the fraction of desorbed polymers in the long chain limit is a step function of $N$, with $f(N)=1$ for $N<N^{*}$ for some $N^{*}$ and $f(N)=0$ otherwise. The constraint (7) in the long chain limit thus takes the form

$\int_{0}^{\infty} \mathrm{d} N \sigma(N) N f(N)=\int_{0}^{N^{*}} \mathrm{~d} N \sigma(N) N=c \varepsilon^{2 / 5}$

where $\sigma(N)=\sigma P(N)$ is the partial grafting density. This defines $N^{*}(\varepsilon)$ as a function that grows monotonously as a function of $\varepsilon$. Hence one would expect that for given value of the adsorption parameter $\varepsilon$, all chains shorter than the threshold value $N^{*}(\varepsilon)$ belong to the adsorbed phase and do not contribute to the residual brush, while all chains with $N>N^{*}(\varepsilon)$ are not affected by adsorption at all. With increasing $\varepsilon$, the part of $\sigma(N)$ that contributes to the residual brush is cut off from below (for $N<N^{*}(\varepsilon)$ ), the total effective grafting density (as associated with the area under the curve) decreases and at the same time, the MMD in the brush is narrowed since shorter chains are completely removed. It is clear that this removal effect is limited by the adsorption capacity of the surface, as well as by the fact that the "infinite chain limit" becomes increasingly questionable for the shorter chains. The self-consistent field calculations presented below will show that the full picture is more nuanced and the preference for short chain adsorption is not absolute.

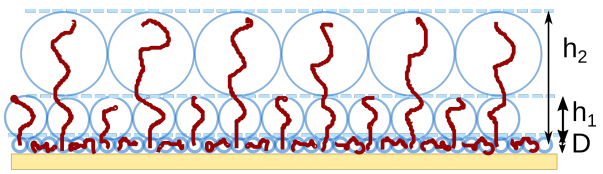

Figure 13. Structure of a partially adsorbed polydisperse brush (here: bidisperse) in the scaling picture. With increasing adsorption strength, the lower layers are gradually soaked up by the adsorption layer (see text).

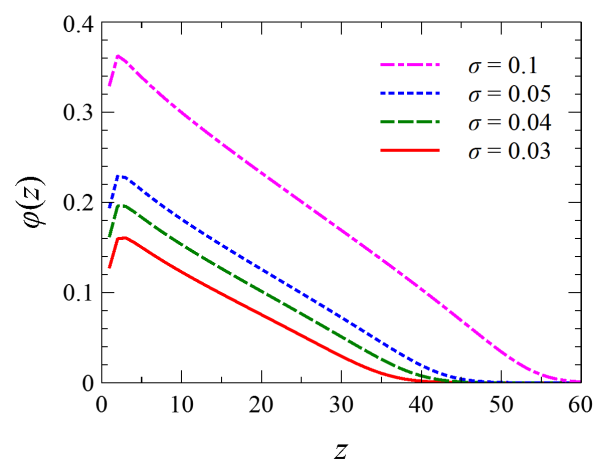

Figure 14. Density profiles (monomer volume fraction) $\varphi(z)$ in a polydisperse brush with "flat" chain length distribution grafted on neutral surface $\varepsilon=0$ at various grafting density $\sigma$, as indicated.

\section{B. Polydisperse brush with flat chain length distribution}

\section{Polydisperse brush with flat chain length distribution grafted onto inert surface}

In order to illustrate the effects of preferential adsorption in a polydisperse brush, we take a very simple MMD shape, namely a uniform distribution. In terms of the number chains of a given length, $n$, per unit area, we define the partial grafting density function

$$
\sigma(n)=\frac{\sigma}{N_{\max }}, 1 \leq n \leq N_{\max }
$$

so that the sum $\sum_{1}^{N_{\max }} \sigma(n)=\sigma$ represents the total grafting density. The mean chain length is given by $\bar{N}=\frac{N_{\max }+1}{2}$ and we take $N_{\max }=199$ for consistency with earlier results. The polydispersity index for this distribution $N_{W} / N_{n}=4 / 3$. Such a chain length distribution is a toy model and was not studied in the literature. To set the background we discuss first the structure of a brush grafted onto an inert non-attractive surface.

Figure 14 shows the monomer density profiles for several values of surface grafting density. It is clear that the brush density profiles decrease almost linearly with the distance from the grafting surface, $z$, except for a small dip in the density very close to the solid surface. Curiously, a similar linear shape of the brush density profile was observed in polydisperse brushes with other forms of the chain length distribution 22, 23, with larger polydispersity index values around $N_{W} / N_{n} \simeq 1.5$. 


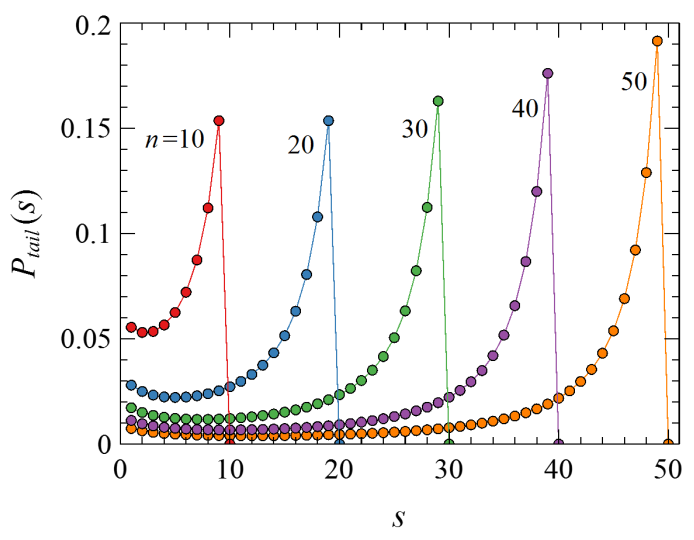

Figure 15. Tail length distributions for several chain length fractions, $n$, (as indicated) in a polydisperse brush with flat chain length distribution grafted onto an inert surface $\varepsilon=0$ at the density $\sigma=0.05$.

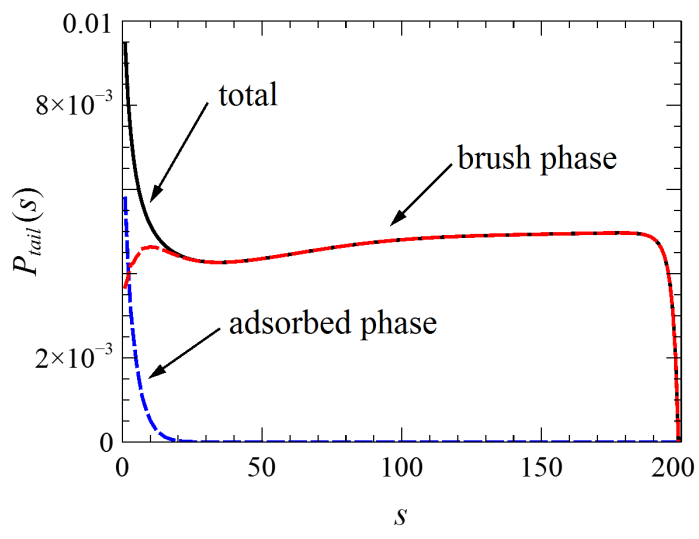

Figure 16. Overall normalized tail length distribution in a the polydisperse brush with uniform MMD grafted onto an inert surface $\varepsilon=0$ at density $\sigma=0.05$, together with its decomposition into two separate contributions from the brush and the adsorbed phases as indicated.

Extending the method of evaluating the relative weights of the adsorbed and the brush phases based on the shape of the tail length distribution, we evaluate these distributions for each chain length in the range from $n=2$ to 200, see Fig. 15. Conformations with tails longer than the position of the minimum are assigned to the brush phase and short tail conformations to the "adsorbed" phase. Although we have no explicit monomer-surface attraction here, we extend the terminology of "adsorbed phase" for the sake of consistency. As mentioned above, this terminology seems counter intuitive but is perfectly compatible with the observation that a considerable number of monomers is in contact with the surface, especially in short chains. The total tail length distribution can be thus presented as a sum of two contributions provided by the two phases, see Figure 16 We are specifically interested in the tail length distribution attributed to the brush phase which turns out to be very close to the flat shape of the MMD.
2. Polydisperse brush with flat chain length distribution, grafted onto an adsorbing surface

In the presence of monomer-surface attraction the brush density profile changes, see Figure 17. With increasing polymer-surface attraction $\varepsilon$, the brush is thinning both in terms of the average density and in terms of thickness, although its nearly linear shape persists; naturally a large density peak appears at the surface due to adsorption. These effects are more pronounced for sparse grafting.

Figure 18 illustrates the effect of preferential adsorption in a brush with continuous polydispersity: the average fraction of adsorbed monomers is displayed as a function of $\varepsilon$ separately for chains of different length, $n$. It is clear that the tendency for adsorption increases continuously as one goes to ever shorter chains.

Now we repeat the procedure of decomposing the total tail length distribution into separate contributions from the adsorbed and the brush phase as discussed in the previous subsection for several values of the adsorption parameter, $\varepsilon$. The idea is to use the brush phase contribution to the tail length distribution $P_{\text {tail }}(s)$ (normalized to give the number of tails of length $n$ per unit area) as an approximation for the $\sigma_{\text {eff }}(n)$ function and therefore an indicator of the effective MMD of the residual brush. This identification is not perfect but solves the problem of an appropriate definition of the MMD of the residual brush (or, alternatively of $\sigma_{\text {eff }}(n)$ ), and works well in the special case of an inert surface as demonstrated above.

The evolution of the tail length distribution in the residual brush normalized per unit area with the increase in the adsorption parameter is displayed in Figure 19. Compared with the naive expectations of the curve being cut off from below as a result of absolute adsorption preference for shortest available chains, the picture is more nuanced. Indeed, the short tails are gradually removed and the area under the curve decreases indicating an overall thinning of the residual brush. On the other hand, longer chains are also partially removed by assuming an adsorbed state. As a result not only the width of the distribution changes but its shape evolves away from a uniform distribution as well.

\section{SUMMARY AND OUTLOOK}

In the present paper, we have examined the properties of polydisperse brushes formed by adsorptionactive chains. We have shown earlier [5] that monodisperse brushes formed by adsorption-active chains demonstrated microphase segregation into a dense adsorbed phase localized close to the grafting surface, and a residual brush phase. The dominant adsorption scenario is such that the chain belongs to one or the other phase as a whole.

As one important result of the present work, we present a novel approach to analysing such situations in detail. Microphase segregation in brushes is 

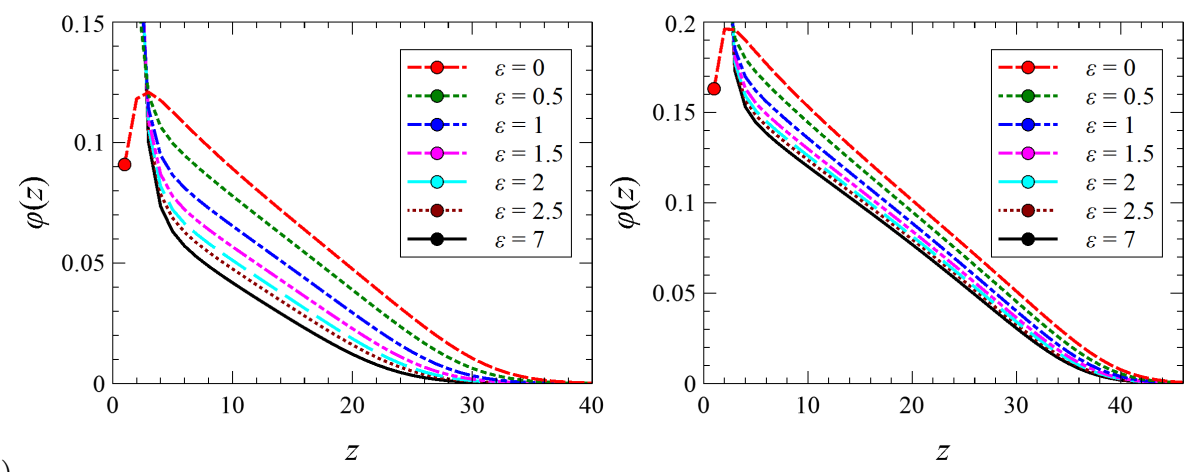

(a)

Figure 17. Density profiles $\varphi(z)$ in a polydisperse brush with uniform MMD at various values of the polymer-surface adsorption energy $\varepsilon$, as indicated ; the grafting density $\sigma=0.02$ (a) and $\sigma=0.04$ (b)

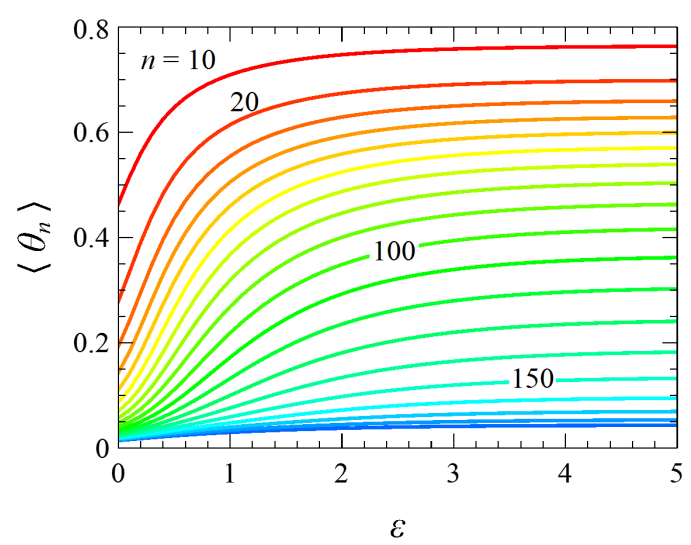

Figure 18. Average fraction of adsorbed monomers in a polydisperse brush with flat chain length distribution grafted at the density $\sigma=0.04$, vs. adsorption energy $\varepsilon$, for chains of different length $n$ as indicated in the graph

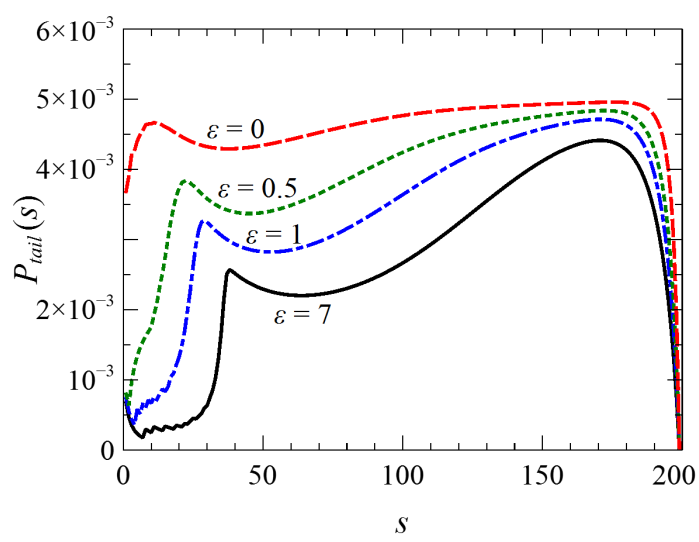

(b)

Figure 19. Tail length distribution in the brush phase in a polydisperse brush with "flat" chain length distribution grafted at the density $\sigma=0.04$, at various values of the polymer-surface adsorption energy $\varepsilon$, as indicated.

a delicate effect that cannot easily be quantified in an unambiguous manner. Here we have introduced a method of assigning different chain conformations to one or the other microphase based on the analysis of tail length distributions. This method gives results consistent with those obtained by direct fitting of the residual brush density profile but has the advantage of avoiding the ambiguity of multi-parameter fitting. Microphase analysis gives a counter intuitive result: Even in the absence of explicit attraction to the surface, one can identify a "phantom" of the adsorbed phase composed by chains with a short tail and a large fraction of monomers in contact with the surface.

Looking at polydisperse brushes, we found that the properties of the residual brush as encoded by its density profile closely resemble that of a monodisperse brush with an effective grafting density that decreases smoothly as the attraction strength goes up. This general picture of an equilibrium between two coexisting phases that can be shifted by changing the adsorption strength is given an additional dimension in the case of polydisperse brushes. We have demonstrated that adsorption from a polydisperse brush exhibits an unexpected effect: Although all chains are chemically identical, shorter chains are adsorbed preferentially. As a result, an increase in the surface affinity parameter is not only accompanied by a reduction in the surface grafting density of the residual brush, but also by a change in the shape of the effective molecular mass distribution. We believe that the effect of preferential adsorption reflects a fundamental property of a polydisperse brush as a reservoir for exchanging monomers with another coexisting phase: monomers belonging to chains of different length are characterized by different effective chemical potentials. Hence brush polydispersity would also affect coexistence with any other condensed phase, not necessarily related to adsorption, examples ranging from brushes with a possibility of liquid-crystalline ordering 24] to polyelectrolyte brushes in external electric field 25].

The data that support the findings of this study are available from the corresponding author upon reasonable request.

\section{ACKNOWLEDGMENTS}

Financial supported by the Russian Foundation for Basic Research through the grant no. 20-5312020 NNIO_a and by the German Science Foun- 
dation through the grant Schm 985/23 is gratefully acknowledged.

[1] N. Ayres. Polymer brushes: Applications in biomaterials and nanotechnology. Polym. Chem., 1:769777, 2010.

[2] M. Motornov, S. Minko, K.-J. Eichhorn, M. Nitschke, F Simon, and M Stamm. Reversible tuning of wetting behavior of polymer surface with responsive polymer brushes. Langmuir, 19(19):8077-8085, 2003.

[3] M. W. Urban. Handbook of Stimuli-Responsive Materials. Wiley-VCH Verlag $\mathrm{GmbH} \& \mathrm{Co}$. KGaA, Weinheim, Germany, 2011.

[4] B. Jaquet, D. Wei, B. Reck, F. Reinhold, X. Zhang, $\mathrm{H}$. $\mathrm{Wu}$, and M. Morbidelli. Stabilization of polymer colloid dispersions with ph-sensitive poly-acrylic acid brushes. Colloid Polym. Sci., 291(7):16591667, 2013.

[5] L. I. Klushin, A. M. Skvortsov, A. A. Polotsky, A. S. Ivanova, and F. Schmid. Polymer brushes with reversibly tunable grafting density. J. Chem. Phys., 154(7):074904, 2021.

[6] R. Descas, J.-U. Sommer, and A. Blumen. Static and dynamic properties of tethered chains at adsorbing surfaces: A monte carlo study. The Journal of Chemical Physics, 120(18):8831-8840, 2004.

[7] R. Descas, J.-U. Sommer, and A. Blumen. Concentration and saturation effects of tethered polymer chains on adsorbing surfaces. The Journal of Chemical Physics, 125(21):214702, 2006.

[8] G J Fleer, M A Cohen Stuart, J M H M Scheutjens, T Cosgrove, and B Vincent. Polymers at Interfaces. Chapman and Hall, London, 1993.

[9] R. C. Advincula, W. J. Brittain, K. C. Caster, and J. Rühe. Polymer Brushes: Synthesis, Characterization and Applications. Viley-VCH, 2006.

[10] Fadi Shamout, Alessandra Monaco, Gokhan Yilmaz, Caglar Remzi Becer, and Laura Hartmann. Synthesis of brush-like glycopolymers with monodisperse, sequence-defined side chains and their interactions with plant and animal lectins. Macromolecular rapid communications, 41:1900459, 2020.

[11] Chaojian Chen, Katrin Wunderlich, Debashish Mukherji, Kaloian Koynov, Astrid Johanna Heck, Marco Raabe, Matthias Barz, George Fytas, Kurt Kremer, David Yuen Wah Ng, and Tanja Weil. Precision Anisotropic Brush Polymers by Sequence Controlled Chemistry. Journal of the American Chemical Society, 142(3):1332-1340, JAN 222020.

[12] R. R. Patil, S. Turgman-Cohen, J. Šrogl, D. Kiserow, and J. Genzer. On-demand degrafting and the study of molecular weight and grafting density of poly(methyl methacrylate) brushes on flat silica substrates. Langmuir, 31(8):2372-2381, 2015.

[13] C. M. Wijmans, J. M. H. M. Scheutjens, and E. B. Zhulina. Self-consistent field theories for polymer brushes: lattice calculations and an asymptotic analytical description. Macromolecules, 25(10):26572665, 1992.

[14] W.M. de Vos and F. A. M. Leermakers. Modeling the structure of a polydisperse polymer brush. Polymer, 50(1):305 - 316, 2009.

[15] P. G. de Gennes and P. Pincus. Scaling theory of polymer adsorption: Proximal exponent. $J$. Physique - Lettres, 44:L-241-L-246, 1983.

[16] P. Grassberger. Simulations of grafted polymers in a good solvent. J. Phys. A: Math. Gen., 38:323, 2005.

[17] L. I. Klushin, A. A. Polotsky, H.-P. Hsu, D. A. Markelov, K. Binder, and A. M. Skvortsov. Adsorption of a single polymer chain on a surface: Effects of the potential range. Phys. Rev. E, 87:022604, Feb 2013.

[18] S. Zhang, S. Qi, L. I. Klushin, A. M. Skvortsov, D. Yan, and F. Schmid. Phase transitions in single macromolecules: Loop-stretch transition versus loop adsorption transition in end-grafted polymer chains. J. Chem. Phys., 148:044903, 2018.

[19] T M Birshtein, Yu V Liatskaya, and E B Zhulina. Theory of supermolecular structures of polydisperse block copolymers: 1. Planar layers of grafted chains. Polymer, 31(11):2185-2196, 1990.

[20] A Halperin. On Polymer Brushes and Blobology: An Introduction. In Y. Rabin and R. Bruinsma, editors, Soft order in Physical Systems. NATO ASI Series, volume 323, pages 33-56. Springer, Berlin Heidelberg, 1994.

[21] G.-L. He, H. Merlitz, J.-U. Sommer, and Ch.-X. Wu. Static and dynamic properties of polymer brushes at moderate and high grafting densities: A molecular dynamics study. Macromolecules, 40(18):67216730, 2007.

[22] S. Qi, L. I. Klushin, A. M. Skvortsov, and F. Schmid. Polydisperse polymer brush: Internal structure, critical behavior, and interaction with flow. Macromolecules, 49:9665-9683, 2016.

[23] L. I. Klushin, A. M. Skvortsov, S. Qui, and F. Schmid. Polydisperse brush with the linear density profile. Polym. Sci. Ser. C, 60(suppl. 1):84-94, 2018.

[24] T M Birshtein and V M Amoskov. Polymer brushes. Polym. Sci. Ser. C, 42(2):172-207, 2000.

[25] H. Merlitz, C. Li, C. Wu, and J.-U. Sommer. Polyelectrolyte brushes in external fields: molecular dynamics simulations and mean-field theory. Soft Matter, 11:5688-5696, 2015. 